\title{
Transversalización de género: un acercamiento al caso de Colombia y una propuesta interseccional ${ }^{*}$
}

DOI: https://doi.org/10.18046/recs.i32.3530

\author{
Gender Mainstreaming: An Approach to the Colombian \\ Case and an Intersectional Proposal
}

\author{
Yira Isabel Miranda-Montero ${ }^{* *}$ \\ Universidad Industrial de Santander (Bucaramanga, Colombia) \\ Priscyll Anctil-Avoine $e^{* * *}$ \\ Université du Québec à Montréal (Montréal, Canadá)
}

Olena Hankivsky ${ }^{* * *}$

University of Melbourne (Melbourne, Australia)

\begin{abstract}
* Este artículo hace parte de los resultados de un proyecto de investigación más amplio titulado "Advancing Equity in Public Policy: An Intersectional Analysis of Gender Mainstreaming in Canada, Sweden, Colombia and the UK", dirigido por la profesora Olena Hankivsky (University of Melbourne) y financiado por el Social Sciences and Humanities Research Council (Canadá). Artículo de investigación recibido el 15.04.2019 y aceptado el 25.03.2020.

** Trabajadora social de la Universidad Industrial de Santander (Bucaramanga, Colombia) y Directora regional de la Fundación Lüvo. Correo electrónico: yiramirandamont@gmail.com ORCID: https://orcid.org/oooo-00o3-0651-0178

*** Candidata al Doctorado en Ciencia política y Estudios feministas de la Université du Québec à Montréal (Montréal, Canadá) y Directora principal de la Fundación Lüvo. Correo electrónico: priscyll.anctil@gmail.com ORCID: https:// orcid.org/oooo-ooo3-3622-3428
\end{abstract}

**** Profesora, University of Melbourne (Melbourne, Australia). Directora, Centre for Health Equity. Correo electrónico: o.hankivsky@unimelb.edu.au ORCID: https://orcid.org/oooo-ooo2-6083-7238 


\section{Cómo citar/How to cite}

Miranda-Montero, Yira Isabel; Anctil-Avoine, Priscyll; Hankivsky, Olena (2020).

Transversalización de género: un acercamiento al caso de Colombia y una propuesta interseccional. Revista CS, 32, 191-219. https://doi.org/10.18046/recs.i32.3530 


\section{Resumen}

Partiendo de los relatos personales y profesionales de 30 mujeres, el presente artículo expone los resultados de investigación respecto a la transversalización de género en Colombia. El objetivo es mostrar su comprensión desde tres diferentes ámbitos - académico, sector ONG e instancias del gobierno-con el fin de analizar las resistencias interseccionales como manera de lograr un cambio estructural en las opresiones vividas en el país. La metodología cualitativa se basa en 30 entrevistas semiestructuradas realizadas en Colombia entre 2017 y 2018, y en el análisis de documentos de políticas públicas. Los resultados aquí presentados se dividen en tres secciones donde se expone la comprensión que se tiene de la transversalización de género, los enfoques diferenciales en que se basó su implementación y las propuestas de las mujeres participantes sobre la interseccionalidad como una forma de lograr un cambio estructural.

\section{PALABRAS CLAVE:}

políticas públicas, transversalización de género, interseccionalidad, resistencias corporales, Colombia

Based on the personal and professional stories of 30 women, this article presents the results of a pioneering research on gender mainstreaming in Colombia. The objective is to understand it in three different areas: the academia, the NGO sector, and the government, in order to analyze the intersectional resistance as a way to achieve structural changes against the multiple oppressions experienced in the country. The qualitative methodology is based on 30 semi-structured interviews conducted in Colombia between 2017-2018, and the analysis of public policy documents. The results presented in this article are divided into three sections that explain the current understanding of gender mainstreaming, the differential approaches on which its implementation was based, and the proposals made by the participating women regarding intersectionality as a way to achieve structural changes.

\section{KEYWORDS:}

Public Policies, Gender Approach, Intersectionality, Embodied Resistance, Colombia 



\section{Introducción}

En un momento de retroceso de los derechos humanos en América Latina frente a una ola conservadora, existe una importante necesidad por interrogar las prácticas que los colectivos feministas, desde el activismo, la academia y los puestos gubernamentales, han propuesto para responder a las opresiones múltiples (Anctil-Avoine; Coenga-Oliveira, 2019). En la teoría feminista interseccional, dichas opresiones múltiples se han entendido como sistemas que ocasionan varias inequidades sociales. Desde esta perspectiva, el análisis de las injusticias sociales debe considerar varios sistemas opresivos, autónomos, pero que no se pueden jerarquizar en el análisis, tales como el sexismo, el racismo, el clasismo o el capacitismo (Crenshaw, 1991; Hill-Collins; Bilge, 2016; Pagé, 2014).

El caso de Colombia se erige como una posibilidad para reflexionar sobre los impactos de la violencia estructural, machista, racista y clasista, sobre el diario vivir de las personas que se identifican como mujeres en el continente. De hecho, la firma del acuerdo de paz entre el gobierno de Juan Manuel Santos y las Fuerzas Armadas Revolucionarias de Colombia-Ejército del Pueblo (FARC-EP) ha dado paso a uno de los programas de posacuerdo más avanzado en términos de enfoques diferenciales de género respecto a los estándares internacionales (Boutron, 2018). Además, es el primer acuerdo de paz, a nivel mundial, que tiene en cuenta las reivindicaciones LGBTIQ+ (Hagen, 2017).

Históricamente, la transversalización de género (TG) se ha convertido en una de las herramientas más importantes de la cooperación internacional y de las políticas de desarrollo ${ }^{1}$ en el marco de las acciones de Naciones Unidas y sus agencias, a partir de la Conferencia de Beijín en el año 1995 (Bacchi; Joan, 2010). En las últimas décadas, las investigaciones han hecho un énfasis importante sobre el análisis de las implicaciones de la TG en las políticas públicas. Sin embargo, poco se ha escrito sobre la evolución de esta hacia una segunda generación de estrategias para abordar los desafíos de las inequidades múltiples (Hankivsky; De Merich; Christofferson, 2019) y la necesidad de reconocer que, como lo argumenta Lozano (2010; 2016), el género intersecta con una gran variedad de opresiones, como la raza y la clase, que moldean la experiencia de la violencia estructural.

En Colombia, diversos grupos locales y regionales han trabajado para implementar los protocolos internacionales de la plataforma de Beijín acerca de la transversalización de género. En el país, dicha TG se ha consolidado en medio de un conflicto

1. Varias autoras feministas decoloniales han criticado este concepto como una imposición de un sistema normativo colonial-moderno (Espinosa-Miñoso, 2016; Rodríguez-Moreno, 2014). Igualmente, Escobar (2011; 2012) propone una crítica posestructuralista a este concepto, abogando por el posdesarrollo. 
armado interno e internacionalizado que ha permitido una normalización de los esquemas violentos (Centro Nacional de Memoria Histórica, 2013), pero también unas prácticas de resistencias a las opresiones múltiples y sistémicas. No obstante, hasta la fecha, no han existido extensivas investigaciones a propósito de la TG en el escenario colombiano. En efecto, muchos estudios se han concentrado en el feminismo decolonial y el antirracismo (Lozano, 2010), el enfoque diferencial (Arteaga-Morales, 2012) o en la implementación de políticas públicas (Esguerra-Muelle; Bello-Ramírez, 2014), pero existe una necesidad de analizar las implicaciones empíricas de la TG como herramienta para responder a la vivencia de múltiples formas de opresiones.

De este modo, el presente artículo busca interrogar estas prácticas a partir de los relatos de 30 personas que se identifican como mujeres y que trabajan en diversos sectores con la TG. Más precisamente, el objetivo es mostrar su comprensión de la TG en tres diferentes ámbitos -el académico, el sector ONG y en las instancias del gobierno- con el fin de analizar las resistencias interseccionales como manera de lograr un cambio estructural en las opresiones vividas en el país. El artículo está dividido entre la parte metodológica y los resultados que, a su vez, se desprenden en tres secciones: una primera, donde se expone la comprensión que se tiene de la transversalización de género; una segunda, que analiza los enfoques diferenciales en los cuales se basó su implementación; y, finalmente, una tercera parte, que incluye la propuesta de las mujeres participantes sobre la interseccionalidad como una forma de lograr un cambio estructural.

\section{Metodología}

La investigación utilizó métodos cualitativos incluyendo la revisión y análisis de documentos de política pública, textos académicos y entrevistas semiestructuradas con representantes activamente comprometidas con el avance de la equidad de género en Colombia. Consiste en 30 entrevistas conducidas con investigadoras y profesoras (10), representantes de organizaciones no gubernamentales (10) y con personas directamente involucradas en la consolidación y ejecución de políticas públicas $(10)^{2}$. De esta manera, la investigación buscó dar unos insumos acerca de las distintas experiencias respecto a la transversalización de género y a las posibilidades de cambio con el enfoque interseccional.

2. Con el fin de conservar el anonimato, las personas entrevistadas están identificadas con su sector de empleo: academia (S), gobierno (G) y sector activista (A). Por ejemplo, una mujer activista de la ciudad de Bogotá será identificada de esta manera: Bogotá, A2. 
Se realizaron las entrevistas semiestructuradas entre los meses de agosto 2017 y mayo 2018 en 9 departamentos de Colombia (Nariño, Cundinamarca, Norte de Santander, Santander, Bolívar, Valle del Cauca, Atlántico, Magdalena, Cauca). El método de reclutamiento de las participantes se dio en dos etapas:1) con la revisión de literatura, se identificaron las personas expertas que trabajan la TG en Colombia, haciendo un primer contacto con ellas; y 2) a partir de este primer acercamiento, se usó una metodología de cadena de afectos (Gómez-Hernández, 2015) para contactar otras personas con dicha experticia. En este sentido, la mayoría de las entrevistas han sido conducidas de manera presencial, en un encuentro bilateral con la persona entrevistada. Sin embargo, algunos de estos ejercicios, por razones de aislamiento geográfico, tuvieron que darse por medio de Skype o por teléfono. Posteriormente, todas las entrevistas fueron transcritas en español por las autoras.

Todas las aprobaciones éticas fueron obtenidas en el marco de la investigación principal, financiada por el Social Sciences and Humanities Research Council de Canadá, "Advancing Equity in Public Policy: An Intersectional Analysis of Gender Mainstreaming in Canada, Sweden, Colombia and the UK". Todas las personas que participaron en las entrevistas han completado y firmado un consentimiento informado y el proceso de recopilación de los datos ha sido anonimizado, con excepción del sector de desempeño laboral de la persona.

Los resultados de las entrevistas fueron analizados partiendo de la premisa de que es posible "contribuir a construir 'espacios' epistémicos insurgentes, transgresores y polifónicos", que dan cuenta de la "diversidad de voces que conviven en un territorio común" (Patiño-Sánchez, 2014: 207), en este caso, Colombia. Así, los relatos de las mujeres se convierten también en un lugar de producción de conocimientos alternos al actual sistema patriarcal, racista y clasista. En este sentido, para la recopilación de los datos, usamos una triangulación entre los documentos de política pública, los artículos académicos y las entrevistas conducidas. El proceso de análisis se dio por medio de Atlas.ti, un programa que permitió caracterizar y organizar la información. En un segundo momento, se construyeron las categorías de análisis y se ordenaron por temáticas; las categorías que se utilizaron en el programa Atlas. ti son las siguientes: interseccionalidad, enfoque diferencial, enfoque poblacional, transversalización de género, opresiones, identidades, política pública, barreras institucionales, barreras estructurales. De ahí, se estableció una red de conceptos vinculando las categorías entre sí (Varguillas, 2006).

Los resultados aquí presentados se dividen en tres secciones donde se expone la comprensión que se tiene de la transversalización de género, ya que las categorías

3. Es importante resaltar que los resultados presentados en este artículo solamente refieren al caso colombiano. 
mujeres, género y enfoque de género se expresaron en el análisis con una interpretación basada en las entrevistas obtenidas. Asimismo, el siguiente segmento se dedica a los enfoques diferenciales en los cuales se basó su implementación, desarrollando las categorías enfoques diferenciales y género. Por último, se encuentra la propuesta de las mujeres participantes sobre la interseccionalidad como una forma de lograr un cambio estructural incipiente, puesto que es desde las márgenes y periferias geopolíticas donde esta se gesta.

\section{Comprendiendo la transversalización de género: algunos apuntes históricos}

\section{Breve recorrido histórico}

En este apartado, proponemos indagar sobre tres puntos:1) un breve recorrido histórico sobre la TG en Colombia; 2) una reflexión sobre la importación de dicho mecanismo desde lo internacional; y 3) los obstáculos nacionales de su implementación.

Primero, es importante resaltar que es la Declaración y Plataforma de Acción de Beijín, en 1995, la que ha dado los lineamientos sobre las políticas constitucionales y jurídicas que tenían que implementarse para la creación de planes y programas que analizaran las cuestiones de género en todos los ámbitos institucionales, políticos, sociales, económicos y culturales en los países miembros. Según el Consejo Económico y Social de las Naciones Unidas (como se citó en Barrig, 2014: 4), la transversalización de género se define "como el proceso de valorar las implicaciones que tiene para los hombres y para las mujeres cualquier acción que se planifique, ya se trate de legislación, políticas o programas, en todas las áreas y en todos los niveles. Es una estrategia [para que mujeres y hombres puedan] beneficiarse de ellos igualmente y no se perpetúe la desigualdad".

Dicho concepto ha venido evolucionando y ha sido adaptado por cada país que lo acoge. En Colombia, se presenta una peculiaridad en la aplicación de la TG, ya que esta se ha hecho en el marco de un conflicto armado que sigue activo, lo cual ha influenciado en gran medida el diseño de políticas públicas, leyes y decretos respecto a la equidad de género. Lo anterior, teniendo en cuenta los enfoques que se fueron creando, dada la necesidad de atención diferencial a las poblaciones víctimas (por ejemplo, la Ley 1448 de 2011).

En los años setenta, el Estado colombiano inició la creación de una estructura institucional dedicada a temas relacionados con las mujeres. Sin embargo, fue solamente hasta el año 1991, cuando se estableció la Constitución Política de Colombia, 
que se dio un giro hacia la importancia de adoptar enfoques sobre la diversidad de las poblaciones del país: así, el Artículo 7 confirma que "El Estado reconoce y protege la diversidad étnica y cultural de la Nación colombiana".

Después, en el año 1992, se creó la primera política social por el Consejo Nacional de Política Económica y Social, el CONPES 2626, con la especificidad de dos grupos de población por su edad y género (Departamento Nacional de Colombia [DNC], 1992). En otras palabras, el comienzo de estas políticas estuvo marcado por realizar intervenciones específicas para las mujeres, por ejemplo, "verificar la calidad de los colegios femeninos e implementar el programa de salud para las mujeres fortaleciendo la salud materna" (DNC, 1992: 16), entre otras acciones.

No obstante, es central mencionar que Colombia ha tenido un acercamiento particular con la TG: debido al conflicto armado interno del país, muchas sentencias, leyes y autos se han reflexionado desde el lugar de las múltiples víctimas de este $^{4}$. Según Serrano Murcia (2014: 51-53), el desplazamiento forzado que se ha dado de forma masiva en Colombia ha llevado a la Corte Constitucional a identificar los riesgos específicos y cargas extraordinarias que recaen sobre mujeres (Auto 092/2008), niños, niñas y adolescentes (Auto 251/2008), pueblos indígenas (Auto 004/2009), afrodescendientes (Auto 005/2009) y personas en situación de discapacidad (Auto 006/2009) víctimas del conflicto armado. Luego, es la Ley de víctimas y restitución de tierras, o Ley 1448 de 2011, que vendrá a especificar aún mejor los enfoques diferenciales a raíz de las victimizaciones que han transcurrido durante el conflicto armado. Así, el Departamento Nacional de Planeación (DNP, 2014: 7), argumenta que:

la implementación de la Ley de Víctimas y de Restitución de Tierras ha exigido un diálogo permanente para garantizar la inclusión del enfoque diferencial, el reconocimiento de los saberes específicos y la coordinación de las instituciones rectoras de política. Esto, en el proceso de fortalecimiento de una visión integral o de interseccionalidad que permita comprender las implicaciones del carácter multidimensional del ser humano y de la convergencia de identidades, situaciones, condiciones y posiciones en una misma persona o grupo.

Así, se puede decir que las discusiones en torno a las múltiples afectaciones en el marco del conflicto armado se inscriben también en un movimiento internacional

4. Es importante resaltar que la TG y su implementación en las políticas públicas no es el resultado únicamente de los textos jurídicos que se produjeron con ocasión del conflicto armado. Nuestro argumento aquí es que los diferentes decretos, leyes, autos y sentencias que históricamente fueron ganancia de la presión de los movimientos sociales para el reconocimiento de los hechos violentos, permitieron avanzar en la conceptualización del enfoque de género en Colombia. 
más amplio hacia una expansión de la comprensión de la TG. Como lo plantean Hankivsky y Mussel (2018: 303), se ha dado un movimiento desde las primeras maneras de ver la TG hacia el desarrollo de una segunda generación de estrategias de transversalización, las cuales tomarían en cuenta las necesidades diferenciadas de las personas y los grupos.

Dos años después de la implementación de la Ley 1448 de 2011, se presentó el CONPES 161 de 2013, como la Política Pública Nacional para la Equidad. En esta política se definió que la "transversalización del enfoque de género es 'la incorporación del análisis de género en las políticas públicas que ayuden a intervenir y transformar las realidades sociales actuales, los procesos culturales de construcción de las identidades de género y la división social y sexual del trabajo"' (Barrig, 2014: 4), garantizándose desde el accionar del Estado. Dicha concepción se apoya en el enfoque diferencial de derechos para "enfrenta[r] la discriminación que sufren las mujeres por el hecho de ser mujeres y considera[r] también, la intersección de otras discriminaciones que afectan a grupos particulares de mujeres" (Barrig, 2014:4). Así, a lo largo de los años, se ha ido comprendiendo la TG en el país, a partir de la noción de enfoque operspectiva de género, es decir, la relación que tiene la transversalización con el enfoque de género es que este último se convierte en la forma de aplicar al primero.

En otras palabras, la política dirigida por la Consejería Presidencial para la Equidad de la Mujer ${ }^{5}$ (CPEM) entiende la transversalización del enfoque de género como "una sola estrategia que se expresa a través de dos canales de concreción: su integración en las políticas y la discriminación positiva, también denominada acción afirmativa, plasmada en intervenciones específicas para mujeres [y nivelar su punto de partida con el de los hombres]" (Barrig, 2014: 6).

De este modo, lo que se ha remarcado en las entrevistas realizadas es que

prevalece mucho el enfoque de igualdad de oportunidades, es decir el poner las acciones afirmativas y el lograr el empoderamiento de las mujeres pero sabemos que eso no fue suficiente y Beijing dijo: transversalicemos. Nosotros todavía no hemos logrado transversalizar todas las políticas públicas (Bucaramanga, S7, comunicación personal, 11.11.2017).

5. La CPEM "es una dependencia adscrita a la Vicepresidencia de la República que propone al Presidente de la República, al Vicepresidente de la República, al Jefe de Gabinete y al Director del Departamento, al Gobierno Nacional y a las Entidades territoriales el diseño de las políticas, planes, programas, proyectos y disposiciones necesarias destinadas a promover la igualdad de género y empoderamiento para las mujeres, así como velar por la coordinación, consistencia y coherencia de las mismas" (CPEM, 2019). 
Lo anterior se debe, en gran parte, a que el género se vinculó y se comprendió, per $s e$, en una equivalencia ontológica con el término mujer. Precisamente, así lo afirman de manera insistente las participantes de esta investigación, desde diferentes lugares y su conocimiento situado sobre la TG en el país. Expresaron, a partir de cargos gubernamentales que "como Estado seguimos viendo el género como un sinónimo de mujer" (Bogotá, G2, comunicación personal, 17.08.2017), confirmando las críticas que se le han hecho a las políticas públicas focalizadas en acciones afirmativas. A pesar de aclarar que son dos canales distintos, los gobiernos siguen haciendo énfasis en la estrategia "que se [dirige] a las mujeres o sectores específicos de ellas para paliar la brecha de desigualdad con los varones" (Barrig, 2014: 5). También se puede citar a las voces en instancias académicas, reiterando que "el enfoque de género empezó explícitamente con ese nombre en la academia: mujeres" (Bogotá, S3, comunicación personal, 21.08.2017). Además, una de las académicas menciona que hoy, en Colombia, no se conocen "estrategias que hayan pensado en vincular en algunos procesos a los hombres, no para que se solidaricen con la causa femenina, sino para que construyan a la par otras realidades" (Bucaramanga, S5, comunicación personal, 12.10.2017).

En su momento, al preguntar por el desarrollo que ha tenido la transversalización de género, se mantuvo la concepción de permanencia en la población mujer. Por ejemplo, las participantes resaltan:

Pienso que sí, se ha avanzado, pero no lo suficiente porque a veces la gente confunde género con mujerismo y eso es un gran problema; a veces se habla de perspectiva de género para las mujeres, pero no se están modificando las brechas y tampoco se trabaja con los hombres. (Valle del Cauca, A5, comunicación personal, 23.10.2017)

Se perdió mucho tiempo en involucrar a los hombres, de haber entendido lo que significaba también la masculinidad, por ejemplo, como una identidad que tenía que ser también cuestionada para pensarse realmente la equidad entre los géneros. (Bucaramanga, S2, comunicación personal, 01.09.2017)

Género ya no es igual a mujeres, sino que también implica a los hombres, a las mujeres y a otras identidades diversas. (Bucaramanga, S7, comunicación personal, 11.11.2017)

\section{Importación de la TG: los mecanismos internacionales}

Ahora bien, es necesario reconocer que los temas de género y sus enfoques devienen de las referencias y obligaciones internacionales acogidas por Colombia con el bloque de constitucionalidad, y que, además, para las participantes "hay un gran problema en cómo se diseñan esos instrumentos, en que no tienen en cuenta las especificidades históricas, políticas, no sitúan sus propuestas" (Bogotá, S4, comunicación personal, 
17.08.2017). De este modo, las mujeres entrevistadas perciben que los instrumentos internacionales de TG no responden a las realidades vividas en lo local, como ha sido anotado por varios estudios (Anctil-Avoine; Mejía-Jerez; Tillman, 2018; Falquet, 2011). Según una participante del sector de la academia:

la política pública en América Latina, y particularmente en Colombia, de enfoque de género está diseñada por los organismos internacionales y para estos es lo mismo Buenos Aires que Cochabamba en Bolivia o Putumayo en Colombia. El enfoque de transversalidad formal de la política pública se hace de manera totalmente descontextualizada. (Bucaramanga, S5, comunicación personal, 12.10.2017)

Sumado a lo anterior, el "tema de género es un cumplimiento normativo por estar a la vanguardia de los pactos internacionales y decir: 'no somos tercermundistas', pero aquí no hay un deseo, una voluntad política real de respetar y garantizar los derechos de las mujeres" (Cartagena, A9, comunicación personal, 22.11.2017). No obstante, a través del Artículo 93 de la Constitución Política, la jurisprudencia, por medio del bloque de constitucionalidad, desde 1995, ha moldeado los "parámetros vinculantes de interpretación de los derechos y deberes protegidos por la norma suprema" (Olaya-Arango, 2004: 80). Tales compromisos se han venido comprendiendo como temas de agenda pública que terminan influenciando la formulación de políticas en este campo.

Al respecto, las críticas sobre cómo se implementó la TG en las políticas públicas del país son expuestas por las participantes al señalar que "la transversalidad se queda en el papel, en el documento de política que está bien escrito, pero luego no se puede implementar" (Bucaramanga, G3, comunicación personal, 09.10.2017). De hecho, es importante resaltar que, por ejemplo, en el análisis de la Política Pública de Mujeres y Equidad de Género de Bogotá, se encuentran fallas debido a la falta de coherencia desde lo estipulado en el documento formal hasta lo puesto en práctica (Barón-Mesa; Muñoz-Eraso, 2016: 104).

Según la experiencia vivida por las participantes de esta investigación, se comprende que el problema de la estrategia TG se mantiene en la decisión de continuar ejecutando las acciones afirmativas sin voluntad política para realizar cambios estructurales, demostrando que "mientras las acciones afirmativas ayudan a mantener los estereotipos de sumisión, no se ha tomado en serio el estudio de la normatividad" (Barranquilla, S8, comunicación personal, 02.04.2018). Al mismo tiempo, las personas entrevistadas enfatizan en: 
lo que ha sido una de las recomendaciones del comité de seguimiento de la CEDAW, que reiteradamente ha señalado la debilidad institucionalidad. El comité ha dicho que Colombia se destaca en sacar normas. Leyes las tenemos por montones, leyes contra la violencia, de equidad, económicas, de cuotas de participación, pero la gran brecha está entre la norma y la realidad. (Bucaramanga, G4, comunicación personal, 13.10.2017)

Esto ha producido el efecto contrario a lo buscado, es decir, no ha permitido una real transversalización, sino una sectorización (Bogotá, G2, comunicación personal, 17.08.2017) o, en su defecto, que solo la idea de transversalizar se vuelva muy etérea (Bogotá, G1, comunicación personal, 16.08.2017). Entonces, en cuanto a la TG y cómo se ha comprendido en Colombia, encontramos que "como en nuestro sistema jurídico la ley está tan lejos del derecho de la costumbre consuetudinaria, tenemos que acortar esa brecha entre la política pública, los discursos, lo jurídico y las realidades cotidianas" (Bucaramanga, S5, comunicación personal, 12.10.2017).

\section{Las barreras nacionales en las estructuras públicas colombianas}

La problemática y la barrera más grande son los funcionarios públicos, porque, según estas lideresas, las mujeres se encuentran con funcionarios de las alcaldías o las secretarías de salud, que desconocen completamente las normas y los derechos de las mujeres (Santa Marta, G5, comunicación personal, 27.11.2017). Reiteradamente, las participantes señalaron que:

encontramos que una de las principales dificultades para las transversalizaciones de género tiene que ver, por un lado, con la voluntad política y el actuar de funcionarios y funcionarias públicas que al no estar sensibilizados pueden limitar la implementación de esta perspectiva de manera transversal, por otro lado, la reducida asignación presupuestal. (Cali, G8, comunicación personal, 06.04.2018)

En efecto, una activista cuestiona: “ ¿cómo nos metemos nosotras a tratar un tema de fondo sobre el empleo y las condiciones laborales de las mujeres, si es que sencillamente nos acosan, nos matan, nos violan?" (Cartagena, A9, comunicación personal, 22.11.2017), en consonancia con lo explicado por Barón Mesa y Muñoz Eraso (2017:103),

las mujeres han sido blanco de vulneración y maltrato físico, verbal y psicológico, principalmente por parte de los hombres que en muchos casos terminan en feminicidios. Esta situación refleja la fuerte problemática en la que han estado inmersas las mujeres colombianas, y se ha convertido en referente importante para que los diferentes Gobiernos formulen y pongan en marcha un corpus normativo para tratar de solucionar esos inconvenientes. 
Lo anterior determina el tipo de acciones que se han trabajado desde la estrategia de transversalización en Colombia, ya que no se ha podido avanzar más en el campo de las políticas públicas con integridad debido a la necesidad existente de proteger la vida, principalmente de las mujeres, ante los altos niveles de violencia de género y de violencia racista (Berlanga-Gayón, 2014; Lozano, 2016). A eso, Berlanga Gayón (2014: 43) añade que "la precariedad de la vida", en el caso de las mujeres racializadas, "tiene que ver con el valor que se les asigna a los cuerpos femeninos racializados, y que se traducen en circunstancias materiales muy concretas: pobreza, explotación y marginación". Esta precariedad de la vida, específicamente de la vida racializada, es uno de los factores que se han criticado en esta investigación, tanto desde su marco internacional como trazando su aplicación en los enfoques diferenciales en Colombia.

Sin embargo, en esta investigación se muestra que los diferentes sectores preocupados por las políticas públicas de mujeres y equidad de género han empezado a preguntarse por todas las expresiones de género que también requieren y exigen atención, análisis, implicación en la tarea de la igualdad social y el reconocimiento de sus derechos humanos. Todo esto, al final, tiene que ver o centrar su atención en las corporalidades. Tal y como lo afirman las participantes, las políticas públicas han fragmentado más a la sociedad en proyectos comprendidos a partir de atención a poblaciones, lo cual fortaleció las barreras burocráticas y las dificultades de las comunidades afectadas, sin permitir transformaciones estructurales en las relaciones de poder.

Lo anterior es producto del intento de aplicación de los enfoques diferenciales en las políticas públicas, los cuales no toman en cuenta el saber militante (Bilge, 2015:26) de las personas que viven las opresiones. Las mujeres son contundentes al decir: "en ese sentido ha sido unitaria [la perspectiva de género], en la medida en que solo se fija en los cuerpos marcados, en los cuerpos femeninos o feminizados; en los cuerpos no hetero" (Bogotá, S3, comunicación personal, 21.08.2017), es decir, en los cuerpos problema. Así, en la práctica, la TG no vincula a toda la sociedad en el compromiso de generar cambios culturales, sino que focaliza: hay una confusión con la ejecución de una política pública con enfoque diferencial cuando lo que se aplica es, en realidad, un enfoque poblacional. Por eso, una de las mujeres entrevistadas explica que:

lo que necesitamos es que todos los sectores de la administración pública realmente tengan políticas, macropolíticas, políticas sociales en donde los sujetos no sean simplemente heterosexuales y cisgeneristas, y además entiendan que las personas de los sectores LGBTI, que serían también sujetos de todas sus políticas, no de unas políticas, son personas al mismo tiempo atravesadas por la raza, por la etnicidad, por la edad, por el capacitismo, por el género, por la sexualidad, por la clase, etc. (Bogotá, S4, comunicación personal, 17.08.2017) 
En este punto, resulta importante explicar cómo la TG se contempla desde un enfoque de género que termina contenido en los enfoques diferenciales para su aplicación.

\section{Percepciones sobre la TG aplicada a través de los enfoques diferenciales}

Como se ha mencionado, dicha estrategia internacional se ha entendido y aplicado en el país desde el enfoque de género que, a su vez, se fortaleció a partir de la interrelación de los enfoques diferenciales ${ }^{6}$ (ED), siendo una característica del actuar colombiano en materia de discriminación positiva en las políticas públicas. Para el DNP (2014: 7), se presentan en categorías o elementos constitutivos como: etario, discapacidad, étnico, género y diversidad sexual. De hecho, "fue el contexto del conflicto armado y los daños padecidos por las víctimas de la violencia los que dieron lugar al reconocimiento de las especiales necesidades de la población víctima en razón de sus particulares características" (Valencia-Mosquera, 2015: 12). Es decir, para paliar las consecuencias del conflicto armado en el país, se crea un enfoque de análisis y atención que posibilite tener un marco en el que se prioricen unas características específicas de las personas.

Precisamente, tanto para la academia como para instancias activistas e institucionales, los enfoques diferenciales se conceptualizan de diversas maneras, entre ellas, la del Ministerio de Protección Social, en conjunto con el Alto Comisionado de las Naciones Unidas para los Refugiados (ACNUR), que exponen que el ED:

busca visibilizar vulnerabilidades y vulneraciones específicas de grupos e individuos específicos, y prioriza acciones de protección y restauración de los derechos vulnerados. Implica: identificar los vacíos y riesgos de protección de cada grupo y desarrollar herramientas para dar soluciones, promover la participación equitativa y planear y ejecutar medidas afirmativas basadas en caracterizaciones sistemáticas para la garantía del goce efectivo de los derechos de los diferentes grupos poblacionales. (ACNUR; Ministerio de la Protección social, 2011: 27)

El concepto comienza a variar sin encontrar un sentido unívoco, provocando percepciones del ED como un "supersancocho (...). Una bolsa, entonces la bolsa diferencial en donde yo puedo ir echando hasta que se vaya a reventar la bolsa.

6. Los enfoques diferenciales suponen que no se cataloguen los sujetos de las políticas públicas en categorías poblacionales; sin embargo, en la práctica, muchas veces las personas deben elegir un sector poblacional con el cual se identifican para recibir el apoyo del Estado. 
Y ahí quedó (...) jel enfoque diferencial quedó en un digiturno!" (Bogotá, G2, comunicación personal, 17.08.2017). Por esta razón, se sostiene la advertencia que refieren varios autores en la actualidad, pues aún se puede identificar la ausencia de un concepto concreto respecto al enfoque diferencial, es decir, una noción clara establecida más allá de su utilidad o su finalidad en el análisis de la discriminación estructural de ciertas colectividades, que, al parecer, nunca ha sido definida en los pronunciamientos emitidos a lo largo de la línea identificada en las decisiones de la jurisprudencia constitucional (Ángel-Cabo, 2012; Franco, 2017), como ente que genera el término para sus análisis.

En esta dirección, Meertens (2002: 25) resalta que un enfoque diferencial por género, edad y etnia no solo debe involucrar las necesidades particulares en términos materiales, sino que, además, debe analizarlas desde una óptica relacional. Esto es, tomando en cuenta inequidades, discriminaciones y exclusiones asociadas a diversos factores interrelacionados, acercándose más a una visión interseccional de la cuestión aplicativa del ED. Así, como lo plantea Montealegre (2011: 10-11):

no todos los logros alcanzados han implicado profundas transformaciones de las relaciones en la vida cotidiana ni en la esfera pública, en y entre los géneros, las etnias, en las identidades sexuales o en las condiciones de salud y de clase, por lo que sigue siendo necesario propender por la incorporación de enfoques diferenciales que abran paso a la realización efectiva de los derechos humanos y al logro de la justicia social.

De este modo, los enfoques diferenciales se han asumido desde una perspectiva muy poblacional y en muchas circunstancias, lo cual se ha traducido en una fragmentación de la comprensión de los problemas (Bogotá, G1, comunicación personal, 16.08.2017), produciendo una atomización en las relaciones sociales. De acuerdo con Franco (2017: 25),

esta categorización de la identidad ha sido la base para la generación de políticas, medidas y enfoques de intervención frente al problema de la discriminación, en una tendencia que ha asumido a los grupos poblacionales como etiquetas en lugar de colectividades en lucha por la reivindicación y el reconocimiento de sus derechos conforme a la complejidad que les es propia.

En el mismo orden de ideas, se inscriben varias de las entrevistadas al convenir que la implementación del enfoque diferencial ha usado la focalización no como un instrumento, sino como un pin (Cali, Bogotá, Barranquilla, Bucaramanga, varios sectores). El mismo efecto ocurre del lado que debe ser garante de la aplicación de la transversalización de los enfoques, ya que en Colombia sigue siendo "un tema 
todavía marginal, y que se asume por parte de algunas personas encargadas de estos asuntos, mas no efectivamente como elementos transversales" (Bogotá, G1, comunicación personal, 16.08.2017).

En cuanto a los enfoques diferenciales como aplicativos de la estrategia de transversalización del enfoque de género, se determina que:

sibien los criterios que alimentan el fundamento del enfoque diferencial están enlazados con nociones como vulnerabilidad, necesidades, diversidad, desigualdad, lo cierto es que todos estos comportan un germen común, que tiene que ver con la discriminación estructural e históricamente identificable respecto a ciertas agrupaciones de personas de la nación colombiana. Esta discriminación a su vez, tiene una relación directa con las particularidades de cada grupo poblacional, que como se ha referido, está marcada por las diferencias identitarias y situacionales asociadas a ellas en función de los efectos de desventaja o privilegio que claramente dependen de las condiciones materiales de acceso a recursos y distribución de la riqueza. (Franco, 2017:33)

En concertación, participantes desde el prisma institucional definen que "falta muchísimo una mirada más política, transformadora, que comprenda que la transversalización del enfoque de género implica que lo que se hace desde las instituciones debería apuntar a una transformación estructural" (Bogotá, G1, comunicación personal,16.08.2017). En este sentido, se puede decir que se fragmentan las acciones estatales en términos de las opresiones vividas; esto significa que una persona que vive múltiples formas de afectaciones por diversas razones se encuentra frente a una situación imposible por elegir la forma de opresión que más le pueda garantizar el apoyo del Estado. Aunque tales apuestas se ven reducidas -y en muchos casos también negadas para soportar el estado de desigualdad e inequidad en el país-, ya existen acepciones desde esas mismas locis, reconociendo que "la transversalización relacionada con la interseccionalidad está avanzando más rápido desde los movimientos sociales porque les está tocando desde abajo, y es una lucha" (Bogotá, G2, comunicación personal, 17.08.2017).

\section{Una resistencia corporal interseccional: pistas de reflexiones}

Cuestionar, en esta investigación, posiciones de poder en el mundo, así como los privilegios de cada persona, es un avance en la decolonización de la misma. Dicho proceso reactiva memorias corporales de poder y resistencia e, incluso, ha inquietado sobre dar a conocer más acerca de los resultados del estudio como una crítica 
a sí misma, a la academia, al activismo y a las relaciones de poder que se ejercen desde las instituciones de gobierno, lugares en los que ya se ubican las mujeres que participaron y quienes se unen en la pluriversidad de voces que han trabajado y aportado en la tarea de transversalizar el género en las políticas públicas del país.

Estos lugares son los cuerpos; los mismos cuerpos de las mujeres que resisten y construyen marcos de actuación para avanzar en las luchas contra las desigualdades epistémicas en su diario vivir. Así, además de analizar el cuerpo como lugar de sufrimiento, se propone ver la corporeidad como un espacio de posibilidades analíticas y prácticas: los cuerpos son potencias (Preciado, 2003). Partir del cuerpo, es también comprenderlo como

un proyecto epistémico que busca trascender la visión euro-occidental del cuerpo que ha conllevado varias consecuencias sobre las corporalidades de las mujeres: por una parte, ha depravado el universo femenino de posibilidades que caen fuera de la "razón masculina" (y muchas veces clasista y blanca) y, por otra parte, ha contribuido al desprecio tanto del cuerpo como de lo femenino. (Anctil-Avoine, 2017: 11)

En los relatos de vida profesional y personal de las mujeres, se ha destacado que, frente a las violencias estructurales, las opresiones vividas en sus cuerpos también se transforman en ejes de contestación contra los marcos impuestos en el ámbito nacional e internacional. Por ejemplo, una mujer académica relata:

decimos: "lo que no pasa por el cuerpo no pasa"; entonces todo tiene que pasar por el cuerpo y de los aprendizajes que tenemos es que las metodologías surgen porque pasan por el cuerpo y así deberían ser las políticas públicas que, si pasan por el cuerpo de una manera positiva y satisfactoria, realmente generan recordación y cambian la vida de las personas. (Bucaramanga S7, comunicación personal, 11.11.2017)

Los cuerpos han sido testigos y víctimas, pero también son los que han aprendido a resistir, a proponer, a crear espacios seguros para que las personas no sigan siendo invisibilizadas por la sociedad. Los cuerpos, los que viven las opresiones, han padecido, pero también han luchado para que las políticas públicas puedan diseñar e implementar formas de prevenir la discriminación o garantizar el derecho a ser reconocidos como seres humanos en interrelación con las demás personas y su hábitat, políticas que deberían ser aplicadas constantemente en nuestra sociedad.

En este sentido, las mujeres que participaron en las entrevistas cuestionaron reiteradamente la implementación de la TG como una imposición externa, desde la agenda internacional, resistiendo también a sus lógicas. De ahí que, como se está 
aplicando para ellas, el enfoque poblacional de género representa un "enfoque que lo que hace es reproducir modelos muy fuertes de clasificación, estigmatización; reproducir modelos jerárquicos de raza, género y clase" (Bogotá, S3, comunicación personal, 21.08.2017). Es un enfoque que fragmenta tanto las identidades como los cuerpos, lo cual conlleva una inadecuación de los protocolos con las realidades encarnadas vividas. La pregunta que surge entonces a la hora de la puesta en marcha de la TG es sobre la capacidad institucional diaria para responder. Así, muchas personas entrevistadas muestran una contrariedad a la hora de pensar en ampliar y mejorar un marco (el ED) que todavía no ha logrado funcionar del todo: el "enfoque de derecho, donde no solamente se tiene en cuenta el sexo sino el género, la cultura, y bueno..., yo no he pensado en cambiarlo porque ni siquiera hemos avanzado en eso" (Bucaramanga, A1, comunicación personal, 30.08.2017).

Por otra parte, las mujeres expresaron que el enfoque diferencial se ha quedado corto en la comprensión de las realidades de un país con un contexto de conflicto armado interno y una cultura machista arraigada hasta en la estructura institucional, dado que no existe una preocupación y sensibilidad por el tema o, en la mayoría de los casos, aún no se entiende de qué se trata la perspectiva diferencial de género.

Teóricamente, la política pública ha presentado la integración del enfoque de género y el enfoque diferencial de derechos humanos para realizar análisis, y proponer planes y programas que tengan en cuenta la interrelación de categorías como clase, etnia, edad y género, además de reconocer que existe intersección de discriminaciones que deben enfrentarse, por lo cual se identifican aspectos del enfoque interseccional en algunos apartes. A su vez, existe una incipiente comprensión y aplicación de un enfoque interseccional que solo se ha comenzado a implementar en ejercicios de formación en políticas públicas de Bogotá y Valle del Cauca (Esguerra-Muelle; Bello-Ramírez, 2014). Desde estas experiencias, y según la Ordenanza Departamental núm. 339/2011 del Valle del Cauca (como se citó en Esguerra-Muelle; Bello-Ramírez, 2014: 23), se entiende que la perspectiva interseccional es "la mirada que reconoce que en las y los sujetos se intersectan distintos sistemas de opresión: sexo-género, raza/racialización/racismo, etnicidad, clase, etario, entre otros, y que, por lo tanto, hay que establecer una visión no poblacional, sino de atención integral y de defensa y protección de derechos teniendo en cuenta la multidimensionalidad de las y los sujetos de la presente política".

Pero, entonces, desde las entrevistas surge la pregunta:

¿Hacemos políticas públicas diferenciadas o hacemos políticas públicas interseccionales? Se necesitan espacios diferenciados en que se piensen las problemáticas, pero a su vez no se aísle de las problemáticas sociales que nos afectan a todos y a todas como las 
políticas de ambiente que nos afectan diferencialmente a las mujeres y a los hombres pero que, como todos, estamos en el mismo espacio vital, nos afecta. (Bucaramanga, S7, comunicación personal, 11.11.2017)

Admitiendo que se ha venido trabajando en Colombia a partir de un ED, las mujeres sienten, de la misma forma, que se debe hacer un giro en la manera de pensar las discriminaciones múltiples. Así, denotan la necesidad de "hacer intersección en las temáticas, no de las poblaciones" (Bucaramanga, S7, comunicación personal, 11.11.2017). La segmentación de las opresiones y de las experiencias vividas conlleva muchas problemáticas a la hora del actuar político y práctico. Una de las participantes menciona que, en su trabajo en una agencia del gobierno que atiende a las víctimas del conflicto armado, la categorización de las personas es un problema que sigue en la práctica:

ahora desarrollando el proyecto, al dar los lineamientos, la Unidad para las Víctimas está dividida en grupos de enfoques, entonces revisaba el lineamiento interseccional, el enfoque de género, el enfoque étnico, el enfoque de niñez y claro era un enfoque interseccional nuevamente de manera segmentada. (Bogotá, G1, comunicación personal, 16.08.2017)

Al contrario, para las mujeres entrevistadas, sus propuestas de comprensión de la interseccionalidad se enmarcan en lo vivido en las diferentes experiencias de la violencia, en el contexto de los territorios y en una geo-corpo-política específica (Coenga-Oliveira; Anctil-Avoine, 2019). Hankivsky y Mussel (2018) sugieren que la interseccionalidad supone, justamente, examinar las relaciones de poder en lugar de aceptar el género como único eje de análisis y de posibilidades para las políticas públicas. Además, subrayan que la interseccionalidad permite operar un movimiento más allá de las categorías de opresión generalmente usadas para considerar diferentes aspectos de dichas opresiones e identidades sociales. En otros términos, se trata de pasar de la visión fragmentada e individual hacia una visión interactiva y sistémica.

Dicha visión se comprende de la siguiente manera: "una mirada interseccional concebida como la necesidad de entender no solamente las afectaciones, sino los sistemas que están detrás y la manera como esos sistemas se imbrican en las afectaciones o vulneraciones" (Bogotá, G1, comunicación personal, 16.o8.2017). La interseccionalidad ya no se comprende de manera categorial-como el enfoque diferencial-, sino como una perspectiva que toma en cuenta múltiples inequidades, opresiones y sistemas de discriminación, los cuales no se pueden jerarquizar (Pagé, 2014). Es decir, el potencial de la interseccionalidad para las políticas públicas es que puede contrarrestar las fragmentaciones categoriales en la atención a víctimas 
de violencias. Una de las participantes apunta: “¿cómo se transversaliza el enfoque en las entidades? Creo que, efectivamente, ir abriéndose a la pregunta desde la interseccionalidad puede ser una manera interesante de aterrizar mucho más este discurso a las realidades concretas de los territorios" (Bucaramanga, S6, comunicación personal, 22.10.2017). Sin embargo, sigue sugiriendo que es un conocimiento superior que aún no se posa en los contextos, concepción que se cuestiona en esta investigación. Más aún, una funcionaria entrevistada expone las ventajas prácticas del enfoque interseccional:

la creación de un mecanismo de participación que es el Consejo Consultivo de Mujeres, para que este mecanismo fuera un escenario de diálogo permanente y para trabajar un criterio participativo y no desde un escritorio. Ahí se da una ampliación y profundización de la comprensión de la interseccionalidad, es decir comprender cómo se cruzan diferentes factores que acentúan y agravan la condición de discriminación de una mujer, si pertenece a una etnia, si tiene una orientación sexual diferente, si se tiene una condición territorial particular... son 21 sectores diversos. Es tal vez de las primeras medidas de orden práctico para comprender la interseccionalidad. (Bucaramanga, G4, comunicación personal, 13.10.2017)

La Figura 1 muestra, según los resultados de la investigación, las posibilidades de la perspectiva interseccional en las políticas públicas. Este enfoque viene ejerciéndose desde los movimientos y luchas de base, desde abajo, paradójicamente, en el lugar donde se piensa que debe aterrizar, porque aún no ha llegado ahí dicho conocimiento. El gráfico, a su vez, muestra un resumen de cómo se ha entendido la transversalización de género en Colombia desde lo estructural, a partir de la utilidad del enfoque poblacional y, luego, del enfoque diferencial que produjo la fragmentación de identidades. En medio de tal fragmentación se originan unas tensiones que al mismo tiempo son las que crean las resistencias a las opresiones desde la antidiscriminación forjada en su mayoría en las márgenes.

En esa línea, las mujeres activistas, académicas y quienes trabajan en entes de los gobiernos, relatan sus experiencias de vida en el trabajo con comunidades, organizaciones, estudiantes, colegas e instituciones. En las respuestas que daban durante la conversación que iba surgiendo, muchas mostraban su desmotivación frente al esfuerzo por lograr un cambio cultural, pedagógico y, fundamentalmente, estructural de las formas en que se mantienen las relaciones sociales; todo se debe a que no se está haciendo seguimiento a la implementación de las políticas y tampoco una evaluación que permita saber qué se puede mejorar o qué no ha funcionado, mucho menos existe voluntad de cambio, según sus declaraciones. 
FIGURA 1 Transversalización de género en Colombia

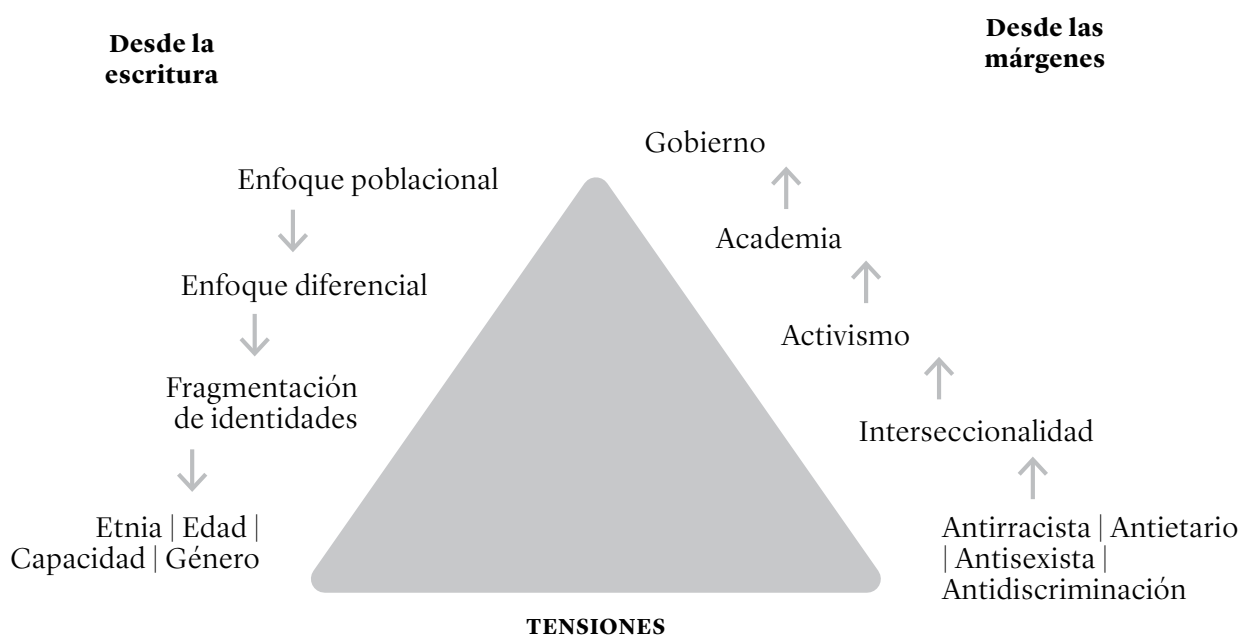

Fuente: elaboración propia a partir de la triangulación Atlas.ti.

Así lo afirma una activista y académica de Buenaventura, Valle del Cauca (A6, comunicación personal, 26.10.2017):

el movimiento pone toda su fuerza en sacar determinada política pública, se mueven en eso, entonces hay mucha movilización, la de feminicidios, la 1257, las de violencias; nos movemos y cuando tenemos la política (...) nos desmovilizamos y cada cual se va a su casa, entonces la política se vuelve un factor de desmovilización del movimiento y a eso le juegan perfectamente los gobiernos.

Además, expresa con firmeza que conceptos como el género y la interseccionalidad presentan una pérdida de sentido o contenido, ya que "cuando estos conceptos, que son conceptos para la transformación, que son conceptos para la liberación, terminan en boca de los funcionarios públicos, ya toca inventarse otros, ya los perdimos" (Buenaventura, A6, comunicación personal, 26.10.2017). Así, la resistencia corporal interseccional se comprende desde las márgenes, como lo propone gráficamente el triángulo sobre la TG en Colombia (Figura 1).

De esta forma, las mujeres entrevistadas consideran que los términos que sirvieron dentro del movimiento social-especifícamente feminista-para la construcción 
de una propuesta de vida social basada en la igualdad, la equidad y el reconocimiento de las múltiples opresiones, se van perdiendo. Es decir, ya han sido utilizados por el discurso hegemónico institucional sin una comprensión y aplicación consciente, política y situada de los conocimientos y saberes que, en últimas, han aportado los feminismos a los derechos humanos. Además, reiteran que, paradójicamente, lo que más obstaculiza bajar a los territorios los reales efectos de las políticas públicas, es la misma estructura gubernamental, puesto que está basada en una cultura aún machista y evidentemente jerárquica.

De hecho, tan solo en 2018 se creó la política pública de género en Buenaventura; es decir, hasta ahora se ha dado un reconocimiento a la existencia de las mujeres negras del país y sus experiencias de vida relacionadas con la pobreza de sus territorios, el color de su piel, lo alejadas del centro de la política geográfica que tenemos $y$, en general, con la desigualdad que caracteriza el nacer en la periferia del país, en las márgenes. Desde que se dio inicio a esta investigación, las mujeres participantes fueron contundentes y precisas al describir el momento político que se avecinaba y por el cual sabían que debían fortalecerse para resistir.

Así, por una parte, las activistas, académicas y funcionarias entrevistadas reconocen las falencias del método colombiano de implementación de la TG, es decir, el enfoque diferencial. Por otra parte, admiten también que la interseccionalidad es un enfoque complejo en su aplicación práctica en el país. Sin embargo, se han realizado aportes importantes a este enfoque desde las periferias de Colombia, acertadamente relacionados con su genealogía en el feminismo negro, tal es el caso del estudio realizado por Lozano (2010:13), en el que afirma que "el concepto género no siempre tomó en cuenta la intersección de 'raza' y clase en su estructuración (Hill, 1998). Y aunque los feminismos son diversos, muchos coinciden en este desconocimiento". Estas mismas conclusiones han sido reafirmadas por las participantes de la presente investigación:

Pese a esas ampliaciones a la mirada de género, esta sigue siendo muy unitaria en un aspecto y es que mira los cuerpos marcados. No mira la masculinidad, por ejemplo, y ahí me baso mucho en las feministas negras. (Bogotá, S3, comunicación personal, 21.08.2017)

Entonces es incompleto aún, están pensando otras identidades, otros cuerpos que han reconocido otras poblaciones LGBTI que son las que viven en las calles, los negros. (Cartagena, A8, comunicación personal, 22.11.2017)

Cómo hacemos para pensar en equidad de género sin pensar en el racismo porque vemos que las más afectadas por el conflicto armado en Colombia fueron las mujeres indígenas y las mujeres afro, campesinas y después las mujeres trans y las lesbianas. (Bogotá, S4, comunicación personal, 17.08.2017) 
[Por ejemplo] las mujeres somos diversas y que el ser diversas implica múltiples opresiones que hay que tener en cuenta entonces que el género no es suficiente como instrumento de análisis. (Buenaventura, A6, comunicación personal, 26.10.2017)

Negar el cuerpo con el que se habita el mundo es el fin de todo proceso de discriminación. En esta investigación los cuerpos de cada participante se volvieron el medio sensible en el que se percibieron muchas realidades, entre estas, las que tienen que ver con la subsistencia de múltiples opresiones. Es decir, en el cuerpo se fusionan varias opresiones, y estas, a su vez, producen condiciones de vida precarias porque:

como lo dice Merleau-Ponty (1962), el cuerpo es nuestro vehículo en este mundo, el lugar donde sentimos y resentimos, el sitio de la lucha política. El cuerpo es un lugar de construcción socio-histórica. Es un lugar de reivindicación y de resiliencia. Es el lugar. (Anctil-Avoine, 2017: 8)

El cuerpo es el lugar donde también se viven significados sociales producidos por las distintas categorías de dominación y desigualdad, como el lugar de nacimiento, la religión, la edad, la diversidad funcional, además de las que ya han sido reiteradamente cuestionadas como el sexo-género, la raza y la clase. Precisamente, lo que proponen las participantes de esta investigación, en diferentes medidas, es lo siguiente: "lo que una mirada interseccional puede hacer es entender que (...) todas las políticas tienen que desmontar las estructuras que generan la desigualdad hacia estas personas que están marcadas racial, sexogenéricamente, por capacidad, por edad, etc." (Bogotá, S4, comunicación personal, 17.08.2017).

De este modo, la investigación ha mostrado la imposibilidad de fragmentar las categorías de identidades y opresión en la transversalización del enfoque de género: dicho método, además de fragmentar la lucha por la justicia social, contradice el trabajo de largo aliento de los grupos y colectivos en los territorios que han llevado a cabo una lucha interseccional encarnada. En este orden de ideas, para algunas de las mujeres entrevistadas, el feminismo decolonial y popular se dibuja como una respuesta a las falencias de la transversalización de género. Según una académica de Bucaramanga (S5, comunicación personal, 12.10.2017):

A mí me resuena mucho el tema [de] los feminismos decoloniales, siento que son una apuesta tanto para América Latina como para Colombia y siento que debemos reconciliarnos con el pensamiento ancestral que ubica la mujer desde la complementariedad y no desde la competencia. 
De los territorios de Colombia, del trabajo de las activistas, funcionarias y académicas, mucho se puede aprender sobre la posibilidad de un feminismo interseccional y decolonial. De esta manera se puede hablar de la interseccionalidad desde las márgenes, en un reconocimiento de las relaciones de poder históricas que mantienen algunos cuerpos en las fronteras de lo inteligible.

\section{Apertura: ¿hacia un enfoque interseccional para la TG?}

Este artículo ha demostrado la peculiaridad de Colombia respecto a la TG. En efecto, su evolución junto a las políticas públicas para hacer frente a las múltiples violencias del conflicto armado ha dado lugar a un trabajo basado en políticas poblacionales. Dichas políticas han fragmentado el actuar acerca de la TG en todas las esferas, sea en lo académico, en el activismo o en los entes del gobierno. Más aún, esta investigación ha mostrado la importancia de trabajar sobre las relaciones de poder que reafirman ciertas separaciones en las luchas contra las inequidades sistémicas, entre estas, la división entre las ciencias, el activismo y la política tradicional. El conocimiento creado en y por los cuerpos está intersectado por diversas formas de opresión, pero es también el lugar desde donde las mujeres entrevistadas construyen sus resistencias en el diario vivir, frente a la violencia estructural.

A modo de conclusión, se pueden trazar 5 ideas principales a las cuales nos convocan las mujeres entrevistadas. Primero, que a raíz de su práctica en términos de TG, se hace fundamental pensar en una pedagogía interseccional para la formación de funcionarias y funcionarios. Según una académica de Barranquilla (S8, comunicación personal, 02.04.2018): "frente a eso tiene que haber una nueva pedagogía que no puede partir de una posición hegemónica y tratar de deconstruir la forma en que se han construido las maneras de conquista y las relaciones de pareja". Segundo, que la transformación cultural contra el machismo y el racismo debe ser uno de los ejes principales de las luchas para poder abrir el camino a la TG. Dicha transformación cultural debe atacar al carácter institucional y naturalizado de la matriz sexo-género-raza (Dorlin, 2014). Tercero, las participantes resaltaron la centralidad de la apropiación de los instrumentos internacionales, pero también su transformación a la realidad colombiana local: "siento que lo fundamental está en la base de la sociedad, en una política del 'voz a voz' de construir otros acuerdos sociales, porque lo de afuera nos llega y nos demoramos mucho en apropiarlo" (Bucaramanga, S5, comunicación personal, 12.10.2017). En cuarto lugar, insistieron en la necesidad de desarrollar mecanismos de ciudadanía inclusiva, apuntando que, en este proceso de TG, las mujeres han olvidado su condición de ciudadana, de sujeta 
política (Cartagena, A9, comunicación personal, 22.11.2017). En quinto lugar, y lo más importante, esta investigación ha demostrado que las prácticas feministas también deben cuestionarse y, entonces, se requiere adoptar un enfoque de interseccionalidad desde las márgenes, para dar cuenta de las realidades vividas en diferentes espacios geopolíticos (Viveros-Vigoya, 2016) que involucran, en relaciones de poder, subalternidad, racismo, clasismo y sus cruces con otros sistemas de opresiones a mujeres y personas de géneros diversos.

Finalmente, esta investigación abre un camino necesario en la relación género-clase-raza, en el sentido que convoca a construir marcos teórico-prácticos sobre la TG, pero desde contextos geosituados. Así, futuras investigaciones podrían abordar algunas cuestiones respecto a lo abordado en este artículo: ¿qué puede aportar un enfoque decolonial a la TG?, ¿sigue siendo pertinente utilizar los marcos internacionales en países como Colombia?, ¿cuáles son las necesidades institucionales, políticas y epistémicas para implementar políticas públicas interseccionales efectivas en Colombia?

\section{Referencias}

Alto Comisionado de las Naciones Unidas para los Refugiados; Ministerio de la Protección Social (2011). Directriz de enfoque diferencial para el goce efectivo de derechos de las personas en situación de desplazamiento forzado con discapacidad en Colombia. Bogotá: Ministerio de la Protección Social.

Anctil-Avoine, Priscyll (2017). "Cuerpos vulnerados, cuerpos violentos": narrativas de mujeres en proceso de reintegración en Bucaramanga, Santander. Bogotá: Corporación Descontamina.

Anctil-Avoine, Priscyll; Coenga-Oliveira, Danielle (2019). Pedagogías decoloniales desde Abya Yala: Desarrollo de teorías feministas a partir de lo vivido. En Enfoque de género para lograr más equidad (pp. 61-75), editado por Louise Lafortune; Vilma Páez-Pérez; Anne Roy. Ottawa: Mapalé.

Anctil-Avoine, Priscyll; Mejía-Jerez, Yuly Andrea; Tillman, Rachel (2018). Gender and the Building Up of Many "Peaces": A Decolonial Perspective from Colombia. En Gender, conflict, peace, and UNSC Resolution 1325 (pp. 231-249). Lanham: Lexington Books.

Ángel-Cabo, Natalia (2012). Enfoque diferencial a la luz de la jurisprudencia constitucional. En Desplazamiento forzado: reflexiones para salir de la encrucijada (pp.520-612), editado por Manuel José Cepeda. Bogotá: Universidad de Los Andes.

Arteaga-Morales, Blanca Inés (2012). Identidades, enfoque diferencial y construcción de paz. Bogotá: Universidad de Bogotá Jorge Tadeo Lozano. 
Bacchi, Carol; Joan, Eveline (2010). Mainstreaming and Neoliberalism: A Contested Relationship. En Mainstreaming Politics: Gendering Practices and Feminist Theory (pp. 396o). Recuperado de http://www.jstor.org/stable/10.20851/j.ctt1t30564.10

Barón-Mesa, Génesis Fernanda; Muñoz-Eraso, Janeth Patricia (2016). Aciertos y fallas en la implementación de la Política de Mujeres y Equidad de Género de Bogotá. Papel Político, 21(1), 101-120. https://doi.org/10.11144/Javeriana.papo21-1.afip

Barrig, Maruja (2014). Transversalizando el género. Trabajo presentado en Encuentro de mecanismos de la mujer y género del orden nacional y territorial, Bogotá. Recuperado de http://www.equidadmujer.gov.co/ejes/Documents/Informe-transversalizacion-generoColombia.pdf

Berlanga-Gayón, Mariana (2014). El color del feminicidio: de los asesinatos de mujeres a la violencia generalizada. El Cotidiano, 184, 47-61.

Bilge, Sirma (2015). Le blanchiment de l'intersectionnalité. Recherches féministes, 28(2), 9-32. https://doi.org/10.7202/1034173ar

Boutron, Camille (2018). Engendering Peacebuilding: The International Gender Nomenclature of Peace Politics and Women's Participation in the Colombian Peace Process. Journal of Peacebuilding o Development, 13(2), 116-121. https://doi.org/10.1080/15423166.2018.146 8799

Centro Nacional de Memoria Histórica (Ed.), (2013). iBasta ya! Colombia, memorias de guerra $y$ dignidad: informe general. Bogotá: CNMH.

Coenga-Oliveira, Danielle; Anctil-Avoine, Priscyll (2019). La corpo-politique: les apports des féministes décoloniales latino-américaines. Féminétudes, 22(1), 108-119.

Consejería Presidencial para la Equidad de la Mujer (2019). Organigrama. Recuperado de https://dapre.presidencia.gov.co/dapre/conozca-el-dapre/organigrama

Crenshaw, Kimberle (1991). Mapping the Margins: Intersectionality, Identity Politics, and Violence against Women of Color. Stanford Law Review, 43(6), 1241-1299. https://doi. org/10.2307/1229039

Departamento Nacional de Colombia (1992). CONPES 2626. Política social para los jóvenes y las mujeres. Bogotá: República de Colombia.

Departamento Nacional de Planeación (2014). Política de atención a víctimas: enfoques diferenciales. Presentación del Subcomité técnico de enfoque diferencial. Bogotá: República de Colombia.

Dorlin, Elsa (2014). La matrice de la race: généalogie sexuelle et colonial de la Nation française. Paris: La Découverte. 
Escobar, Arturo (2011). Encountering Development: The Making and Unmaking of the Third World. Princeton: Princeton University Press.

Escobar, Arturo (2012). Más allá del desarrollo: postdesarrollo y transiciones hacia el pluriverso. Revista de Antropología Social, 21, 23-62.

Esguerra-Muelle, Camila; Bello-Ramírez, Jeisson Alanis (2014). Interseccionalidad y políticas públicas LGBTI en Colombia: usos y desplazamientos de una noción crítica. Revista de Estudios Sociales, 49, 19-32. https://doi.org/10.7440/res49.2014.02

Espinosa-Miñoso, Yuderkys (2016). De por qué es necesario un feminismo descolonial: diferenciación, dominación co-constitutiva de la modernidad occidental y el fin de la política de identidad. Solar, 12(1), 141-171.

Falquet, Jules (2011). Les «féministes autonomes» latino-américaines et caribéennes : vingt ans de critique de la coopération au développement. Recherches féministes, 24(2), 39-58. https://doi.org/10.7202/1007751ar

Franco, Antonio (2017). Axiomas e intersecciones: del enfoque diferencialy los derechos humanos en Colombia [Tesis de maestría]. Universidad Nacional de Colombia, Bogotá, Colombia.

Gómez-Hernández, Esperanza (2015). Investigación decolonial desde el Trabajo Social. Recuperado de http://www.fts.uner.edu.ar/secretarias/academica/catedras_en_linea/ tfoi/2017/Trabajo\%2oSocial\%2odecolonial\%2oEsperanza\%2oGomez-Hernandez\%2O \%20octubre\%2O2015\%2O(1).pdf

Hagen, Jamie J. (2017). Queering Women, Peace and Security in Colombia. Critical Studies on Security, 5(1), 125-129. https://doi.org/10.1080/21624887.2017.1294835

Hankivsky, Olena; De Merich, Diego; Christoffersen, Ashlee (2019). Equalities 'Devolved': Experiences in Mainstreaming Across the UK Devolved Powers Post-Equality Act 2010. British Politics, 14(2), 141-161. https://doi.org/10.1057/s41293-018-00102-3

Hankivsky, Olena; Mussell, Linda (2018). Gender-Based Analysis Plus in Canada: Problems and Possibilities of Integrating Intersectionality. Canadian Public Policy, 44(4), 303-316. https://doi.org/10.3138/cpp.2017-058.

Hill-Collins, Patricia; Bilge, Sirma (2016). Intersectionality. Cambridge: Polity Press.

Lozano, Betty Ruth (2010). El feminismo no puede ser uno porque las mujeres somos diversas. Aportes a un feminismo negro decolonial desde la experiencia de las mujeres negras del Pacífico colombiano. La manzana de la discordia, 5(2), 7-24.

Lozano, Betty Ruth (2016). Violencias contra las mujeres negras: neo conquista y neo colonización de territorios y cuerpos en la región del Pacífico colombiano. La manzana de la discordia, 11(1), 7-17. 
Meertens, Donna (2002). Encrucijadas urbanas. Población desplazada en Bogotá y Soacha: una mirada diferenciada por género, edad y etnia. Bogotá: ACNUR. Recuperado de http:// bdigital.unal.edu.co/47248/

Montealegre, Diana María (2011). Módulo 3.1: enfoques diferenciales de género yetnia. Recuperado de http://www.bivipas.unal.edu.co/bitstream/10720/415/24/D-222-PIUPC-P24-249.pdf

Olaya-Arango, Mónica (2004). El bloque de constitucionalidad en la jurisprudencia de la Corte Constitucional colombiana. Precedente. Revista Jurídica, 79-102.

Pagé, Geneviève (2014). Sur l'indivisibilité de la justice sociale ou Pourquoi le mouvement féministe québécois ne peut faire l'économie d'une analyse intersectionnelle. Nouvelles pratiques sociales, 26(2), 200-217. https://doi.org/10.7202/1029271ar

Patiño-Sánchez, Marisol (2014). La decolonialidad, el buen vivir y el respeto a la diversidad en la construcción de conocimiento, formación e investigación en Trabajo Social. En Diversidad y decolonialidad del saber en las Ciencias Socialesy el Trabajo Social (pp. 205-220), editado por Esperanza Gómez; Marisol Patiño; Esterla Barreto; Francisco González; Jessenia Rivera; John Mario Muñoz...; Martín Román. Medellín: Pulso \& Letra.

Preciado, Paul Béatrice (2003). Multitudes queer. Notas para una política de los "anormales". Multitudes. Revue politique, artistique, philosophique, 12. Recuperado de http://www. multitudes.net/category/l-edition-papier-en-ligne/multitudes-12-printemps-2003/

Rodríguez-Moreno, C. (2014). Mujer y desarrollo: un discurso colonial. El Cotidiano, 184, 31-37.

Serrano-Murcia, Adriana (2014). Enfoque de género en el proceso de DDR. En Desafíos para la reintegración: enfoques de género, edad y etnia (pp. 68-115), editado por Centro Nacional de Memoria Histórica. Bogotá: CNMH.

Valencia-Mosquera, César Augusto (2015). Limitaciones del principio de enfoque diferencial en la reparación a víctimas del conflicto armado interno en Colombia. Acercamiento a la situación de las mujeres afrodescendientes. Recuperado de http://bibliotecadigital.usbcali.edu.co/ bitstream/10819/3148/1/Limitaciones_principio_enfoque_valencia_2015.pdf

Varguillas, Carmen (2006). El uso de atlas.Ti y la creatividad del investigador en el análisis cualitativo de contenido upel. Laurus, 12 (Número extraordinario), 73-87.

Viveros-Vigoya, Mara (2016). La interseccionalidad: una aproximación situada a la dominación. Debate Feminista, 52, 1-17. https://doi.org/10.1016/j.df.2016.09.005 